\title{
Data Analysis and Modeling of Chilled Water Loops in Air Conditioning Systems
}

\author{
Zhida Zhao, Nanyang Yu, Tao Yu, and Haofei Zhang \\ School of Mechanical Engineering, Southwest Jiaotong University, Chengdu 610031, China \\ Correspondence should be addressed to Nanyang Yu; rhinos@126.com
}

Received 20 November 2016; Revised 10 February 2017; Accepted 22 February 2017; Published 13 March 2017

Academic Editor: Marco Pizzarelli

Copyright (c) 2017 Zhida Zhao et al. This is an open access article distributed under the Creative Commons Attribution License, which permits unrestricted use, distribution, and reproduction in any medium, provided the original work is properly cited.

\begin{abstract}
Artificial neural network has been widely used in air conditioning systems as an effective method for predicting parameters, and the accuracy of ANN model relies on training data and network structure. In order to increase the quality of chilled water loops model, this paper develops an optimal data processing algorithm combining Kalman filtering with particle swarm optimization to compensate for uncertain factors and disturbances of collected data from the case building and establishes the nonlinear variation trend database. Based on Elman and BP neural networks, this paper proposes the improved network structures to avoid the local optimum predicted value of chilled water loops and increase data training speed. Simulation results show that this algorithm improves the data accuracy of current percentage (CP) of chillers and chilled water temperatures $12 \%$ and $9 \%$. Compared with Elman and BP models, mean absolute errors of CP improved models are improved $24.1 \%$ and $10.3 \%$, and mean squared errors of water temperature improved models are improved 5.2\% and 4.8\%. For the purpose of energy conservation control in air conditioning systems, this work has an application value and can be used for predicting other parameters of buildings.
\end{abstract}

\section{Introduction}

With the development of artificial pattern recognition and intelligence technology, the information construction of air conditioning systems has been improved. Numerous sensors have been installed in energy management and control system (EMCS), and data classifications are becoming more detailed. When measured data are transmitted to servers using communication technology, it will produce great value. How to use these huge data to resolve the management and optimal configuration problems for the limited energy resources is always the hot topic, which depends on the support of the relevant data processing and an analysis tool. Recently, data analysis technology has been applied to the heating, ventilation, and air conditioning (HVAC) systems. For instance, Chang et al. [1] developed an effective data acquisition and air conditioning control system using Labview; it could realize the real-time data acquisition as well as the data transmission, processing, and display. In addition, this system could save a lot of manual operations and material resources. Wang et al. [2] analyzed the information between the supply air temperature and the other variables acquired from an air conditioning system and presented a data preprocessing and association scheme. Piette and Hannah [3] designed a performance-monitoring tool using data storage and Internet; it could get more high-quality timeseries data for performance analysis. The results showed that this tool facilitated the detection and the diagnosis of energy and other performance problems in buildings. Hou et al. [4] developed a strategy based on the data analysis method to detect and diagnose sensor faults in HVAC systems. Real test results from HVAC systems showed that this strategy could identify temperature sensor faults of the supply chilled water and the return chilled water. Doubtlessly, data quality is the precondition for increasing the analytical efficiency and the database category. Furthermore, it needs an advanced data preprocessing scheme.

Research about the modeling of air conditioning systems can be traced back to the 1930s, and the development was accelerated after the theory of intelligent air conditioning was presented in 1990s [5]. Generally, the evolution of air conditioning system model can be divided into three stages according to different principles, as shown in Figure 1. Physical model is the initial one that describes the heat transfer 


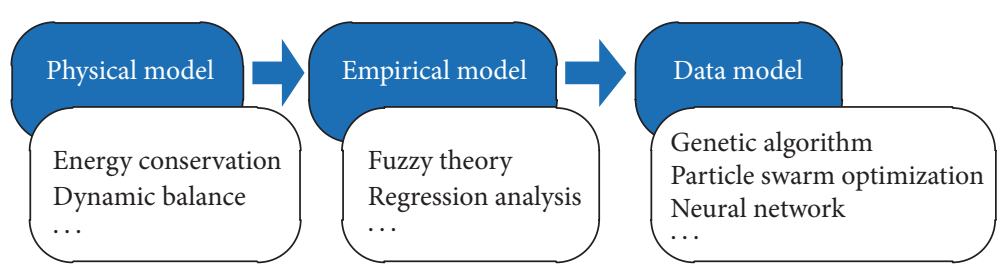

FIGURE 1: The development process of air conditioning model.

process or physical property of components using energy conservation and dynamic balance theories [6]. For instance, Zhang et al. $[7,8]$ discussed the physical structure and the operating characteristic of a chiller using partial differential equation; this model reasonably predicted the chiller transient performance. Whalley and Abdul-Ameer [9] indicated that the air conditioned room model was similar to the circuit resistance and capacitance in electrical system. They introduced the room temperature and the ventilation equipment model and analyzed the response. It is undeniable that physical model can effectively express the intrinsic characteristic of components in air conditioning systems, but many external nonlinear disturbances and phenomena cannot be accurately described. Therefore, many engineers have adopted the optimized or simplified scheme to get the satisfactory performance. He et al. [10] developed a simplified thermal model for predicting the cooling load and the thermal environment in buildings. Compared with other building simulation tools, test results showed that root mean square values of operative temperature and cooling load were below $1 \%$ and $12 \%$. Thosar et al. [11] designed a nonlinear control system for various air conditioning plants to reduce the building energy consumption, and this system was compared with a PI control scheme. The results showed that the reduced model was chosen to ensure the mathematical tractability, and optimal performances in terms of comfort and energy were obtained using this method. However, empirical model often amends many performance coefficients of description equation based on engineers' experience. For instance, Yiu and Wang [12] developed a multiple autoregressive moving average exogenous model for air conditioning system and analyzed the difference in performances when the system model adopted SISO or MIMO structures. The results showed that the predicted values were significantly closer to the measured values. $\mathrm{Xu}$ et al. [13] formulated a real-time dynamic load model for the terminal equipment. In their study, the experimental results showed that the empirical model had the well forecast effect, and more effective energy saving control strategy could be obtained using this model. Nowadays, air conditioning systems have entered into "Data Time", and data model has emerged. Data mining and intelligent algorithms are able to provide a new development trend for the modeling of air conditioning systems $[14,15]$.

Air conditioning systems contain many subsystems, including water system, ventilation system, air supply system, and control system. Among them, the chilled water loops (CWL) are most complicated, and it is difficult to accurately describe this arbitrary component. In multitudinous modeling tools, artificial neural network (ANN) has been widely used as the representative of intelligence algorithm and plays an important role in the data processing. Engineers can get well predicted data according to the network analysis, without knowing the detailed structure about the component [16]. Therefore, ANN and CWL can be combined together. In this field, Frey et al. $[17,18]$ designed the ANN model to describe the chiller performance equation; results showed that the predicted temperature was very close to the test temperature. Hou et al. [19] combined ANN with rough set theory to predict the load of an air conditioned room; results showed that the actual error was within 4\%. Using ANN with 6-6-9-1 structure, Manohar et al. [20] presented a steady state double effect absorption chiller model to optimize the coefficient of performance (COP). The predicted COP had an error of $\pm 1.2 \%$. Zhao et al. [21] built a screw chiller mode using the polynomial ANN, which provided a method to improve the part-load energy efficiency. In their study, the predicted performance by the chillers model was better than existing ones in the literature. Facticity and accuracy are the targets in the air conditioning system model and also decide the performance of the control system. According to these investigations, ANN has the superior performance for data modeling. Unfortunately, if there is any serious nonlinear influence hiding in the collected data, the output value may fall into the local optimal solution. Meanwhile, the process of the collected data will be influenced by multitudinous uncertain and nonlinear factors. Thus, it is difficult to predict the future state.

For taking advantage of measured data and building more precise prediction model, this paper develops an optimization data processing scheme combining Kalman filtering with particle swarm optimization (KPOS) to improve the accuracy of measured data and analyzes the data variation trends for establishing the nonlinear variation trend database (NVTD). Meanwhile, this paper adds evaluation (Eva) layers which are used to update the weight calculation and constrain the boundary conditions based on NVTD in the Elman and BP networks. Finally, this paper predicts the current percentage (CP) of two chillers and chilled water temperatures in a case building using the improved model as a prediction example. The results show that the authenticity of the improved model is enhanced effectively, and locally optimal solution can be avoided using this method.

\section{System Descriptions}

The case building is located in Shenzhen, China. This building was reconstructed into ice storage air conditioning system in 2009, where office area adopts fan coil system, and 
shopping area (opening hours are from 9:00 am to 22:00 pm) adopts all air systems. EMCS includes 34 temperature monitoring points and $2 \mathrm{CP}$ monitoring points as shown in Figure 2. About 2 years' data are collected to expound the data processing scheme and improved models. Some details are presented as follows.

(i) Architecture (5 floors, $30000 \mathrm{~m}^{2}$, shopping and office)

(ii) HVAC system (ice storage air conditioning system)

(iii) Chiller (two screw chillers (total power: $258 \mathrm{~kW}$ ), cooling capacity (air conditioning): $1436 \mathrm{~kW}$, cooling capacity (ice storage condition): $859 \mathrm{~kW}$ )

(iv) Data acquisition

(1) Sample interval: $0.5 \mathrm{~h}$

(2) Total data records: 1345975

(3) Data for modeling: CP, temperatures of chilled water: supply and return water temperatures (TCHWS and TCHWR)

\section{Methodology}

3.1. Data Processing Scheme. The quantitative analysis about limited energy management and optimal configuration in CWL depends on the accuracy collected values (CVs) from EMCS and the mathematical model. Due to the interference and the noise which are the primary uncertainties in measurements when recording the data, missing and abnormal values (MAVs) are unavailable. If MAVs have a serious effect on the prediction model, error between the predicted value and the true value will be increased. Therefore, the observed value cannot be regarded as the real value. For obtaining ideal real values (IRVs), this paper adopts Kalman filtering [22] and particle swarm optimization [23] (KPSO) algorithms to compensate for MAVs.

Kalman filtering algorithm calculates the optimal estimated value from the collected data; state equation and observation equation of collected data can be described using the following:

$$
\begin{aligned}
S_{t}(k) & =M_{s} S_{t}(k-1)+\Gamma B(k-1), \\
O_{b}(k) & =M_{m} S_{t}(k)+F(k) .
\end{aligned}
$$

The deviation of collected data can be calculated using

$$
D(k \mid k-1)=D(k-1)+Q .
$$

The deviation gain can be calculated using

$$
G_{(\mathrm{CP}, T)}=\frac{D(k \mid k-1)}{(D(k \mid k-1)+R)} .
$$

Since the design value is used as IRVs, the deviation gain should be always within the range of $[-0.1,0.1]$. The estimated value can be calculated using

$$
\begin{aligned}
T_{\mathrm{IRV}}(k) & =T(k-1)+G_{T}(T(k)-T(k-1)), \\
\mathrm{CP}_{\mathrm{IRV}}(k) & =\mathrm{CP}(k-1)+G_{\mathrm{CP}}(\mathrm{CP}(k)-\mathrm{CP}(k-1)) .
\end{aligned}
$$

TABLE 1: Summary of water temperature data.

\begin{tabular}{lcc}
\hline Start time & End time & Quantity \\
\hline 2014-07-22, 23:30 & $2014-07-25,23: 30$ & 286 \\
$2014-07-25,23: 30$ & $2014-07-28,13: 30$ & 250 \\
$2014-07-28,14: 00$ & $2014-08-15,00: 30$ & 1676 \\
$2014-08-15,01: 00$ & $2016-02-27,20: 00$ & 18251 \\
$2016-02-27,20: 30$ & $2016-05-27,15: 00$ & 8450 \\
$2016-05-27,15: 30$ & $2016-06-21,14: 30$ & 2396 \\
$2016-06-21,15: 00$ & $2016-08-20,04: 30$ & 5716 \\
\hline & & 37025 \\
\hline
\end{tabular}

The deviation of IRV will be updated using

$$
D_{\mathrm{IRV}(\mathrm{CP}, T)}(k)=\left(1-\mathrm{G}_{(\mathrm{CP}, T)} M_{m}\right) D(k \mid k-1),
$$

where $k$ is sampling instant. The important variables in Kalman filtering are $Q$ and $R$, which are always obtained based on the experience of designers and values are confirmed. However, the actual systems are dynamic; data processing needs to update $Q$ and $R$. Then the PSO algorithm is adopted for searching the optimal values of $Q$ and $R$. In this algorithm, every particle has a fitness value; it can be calculated using

$$
J=\sum_{i}^{n}\left[\left\{\left|T_{\mathrm{CV}}, \mathrm{CP}_{\mathrm{CV}}\right|\right\}-\left\{\left|T_{\mathrm{IRV}}, \mathrm{CP}_{\mathrm{IRV}}\right|\right\}\right]^{2} .
$$

In the iteration, the position of particle is updated using

$$
\begin{aligned}
& v_{i}^{d}=\lambda v_{i}^{d}+c_{1} r_{1}\left(p_{i}^{d}-x_{i}^{d}\right)+c_{2} r_{2}\left(P_{i}^{d}-P_{i}^{d}\right), \\
& x_{i}^{d}=x_{i}^{d}+\alpha v_{i}^{d},
\end{aligned}
$$

where $i=[1 \sim 30] ; p_{i}$ is the best position of particle; $P_{i}$ is the best position of swarm. Figure 3 shows the flowchart of KPOS; it starts with the initial sample data and optimizes MAVs using the Lagrange interpolation until the output value meets the requirements. The observation data $\left(O_{b}(k+1)\right)$ is updated using $S_{t}(k)$ and $D_{\text {IRV }}(k)$ as well. Tables 1 and 2 show the summary of water temperature and chillers energy consumption data. The CVs are inconsecutive because of interference and uncertain factor. For instance, when the data of 1 \# chiller water temperature in 7 days is analyzed, $Q$ is 0.093 and $R$ is 0.165 through KPOS algorithm. Figure 4 shows the comparison between CVs and IRVs. Obviously, MAVs still exist in preprocessing data and the average errors for supply and return chilled water temperatures are $0.67^{\circ} \mathrm{C}$ and $0.43^{\circ} \mathrm{C}$, respectively. Apparently, this method improves the authenticity of the measured data and avoids MAVs effectively. The observed value will be replaced by IRVs when the process of data modeling is executed.

3.2. Nonlinear Variation Trend Database. Data collected from EMCS represents the superficial features of air conditioning systems, so it is necessary to excavate the variation hiding data. Meanwhile, load areas are always influenced by uncertain interference factors, leading to the inevitable 


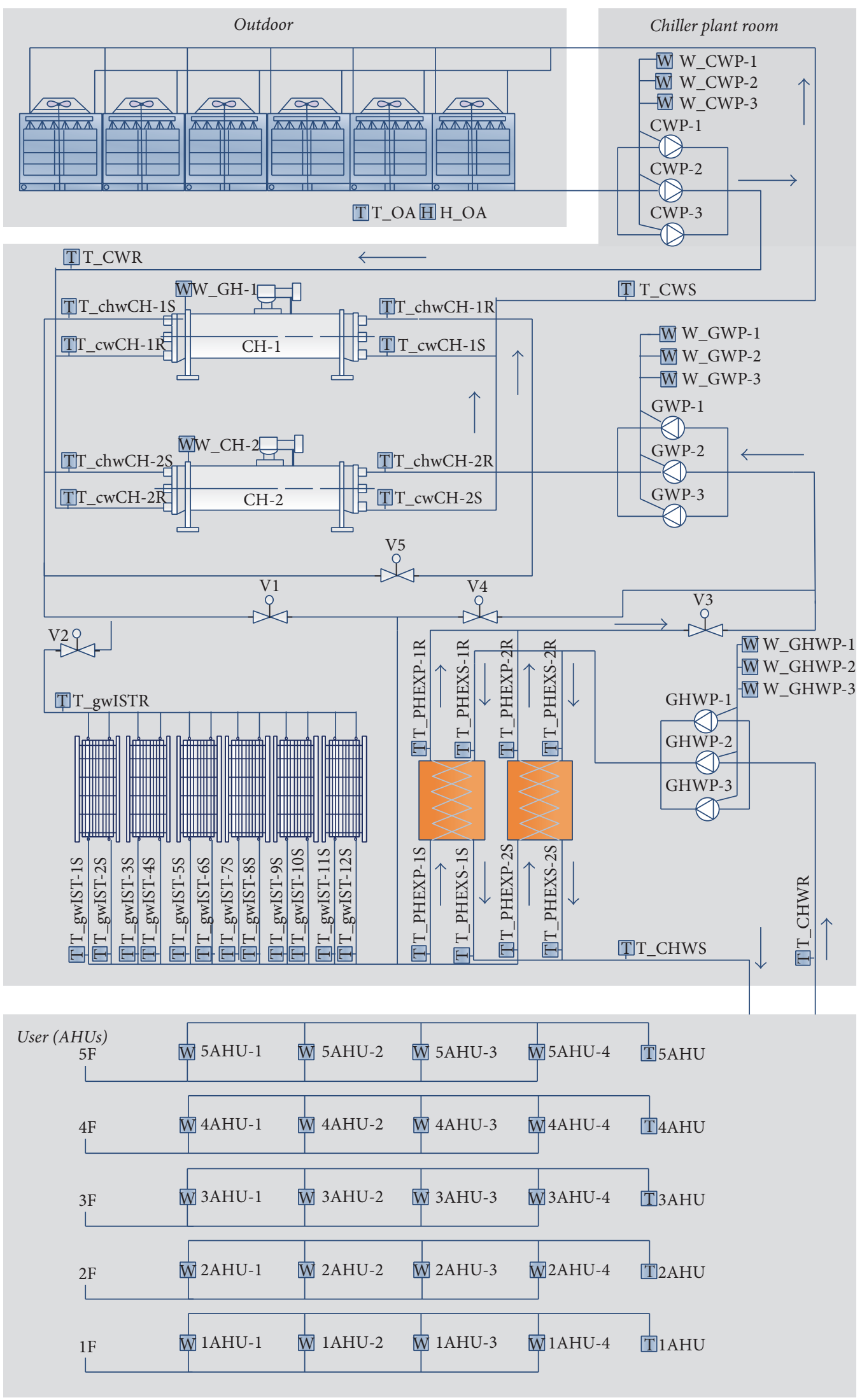

FIGURE 2: The arrangement of MPs in EMCS of a case building. 


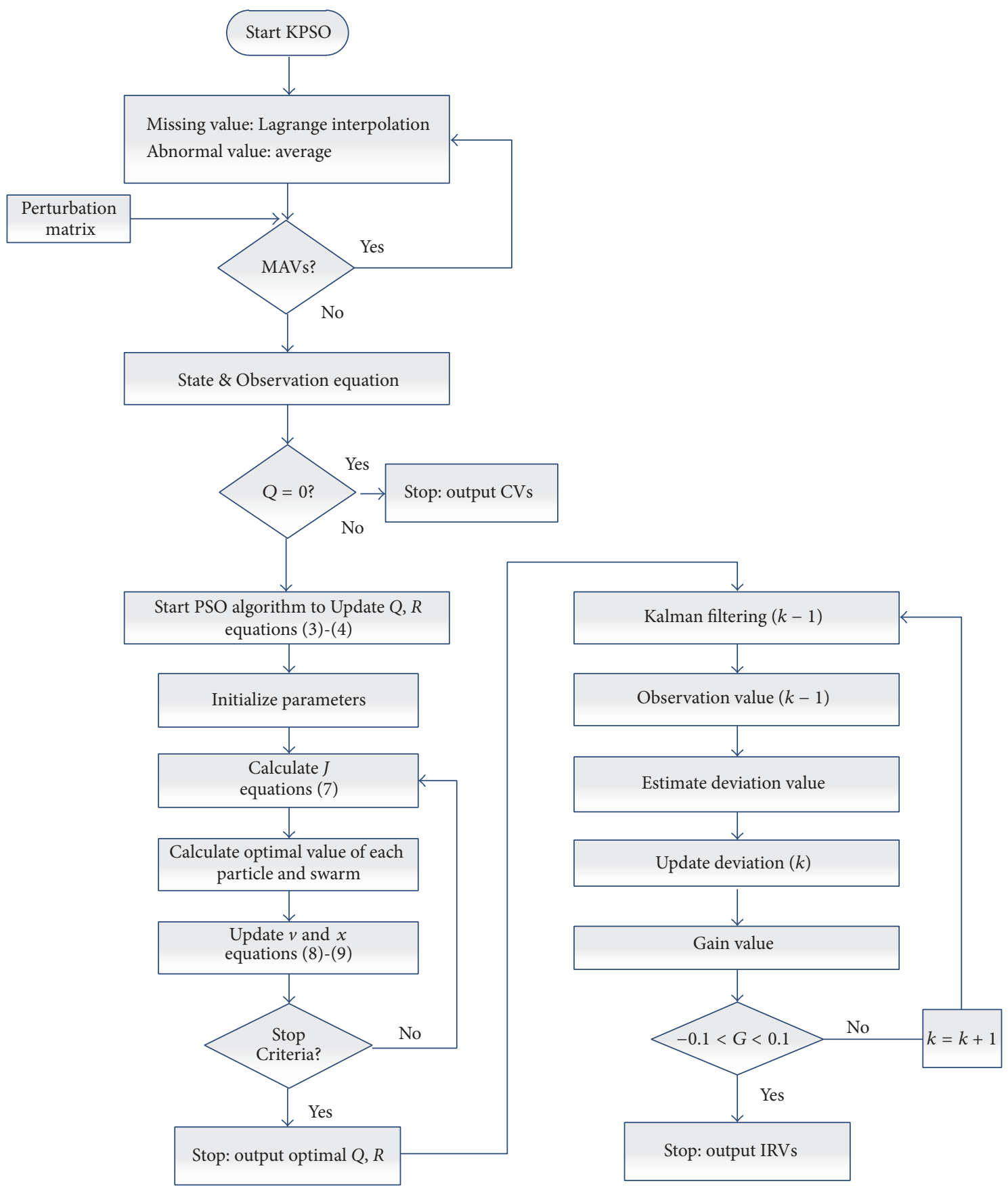

FIGURE 3: Flowchart of KPOS for data processing.

nonlinear phenomena in the input and output places of CWL. In this work, for analyzing the operation of chillers and chilled water temperatures, the supply chilled water temperatures are regarded as input and return chilled water temperatures are regarded as output. CP of chillers is used to illustrate the operation characteristic. To make full use of collected data, the variation trend is divided into two directions, as shown in Figure 5. Similar to the automatic control theory, the ordinate represents the sample data in one day ("hysteresis of water temperature") while the abscissa represents the sample data in a corresponding cycle ("chaos of water temperature"), and these trends are nonlinear. The new database (NVTD) is used for establishing evaluation layer in the process of modeling.

3.2.1. Abscissa: Chaos of Water Temperature. The sample data at the same time of different days is obvious due to the influence of equipment working condition, random employee turnover, and variability of outdoor temperature. So the trend value of this phenomenon can be defined as the 


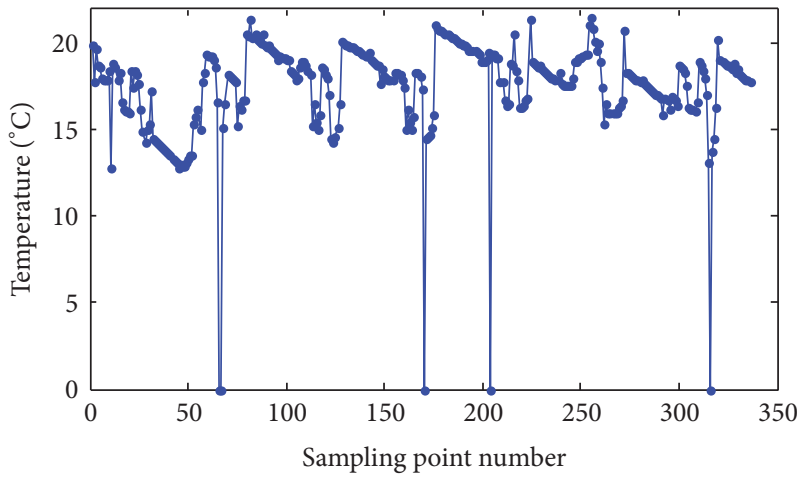

(a)

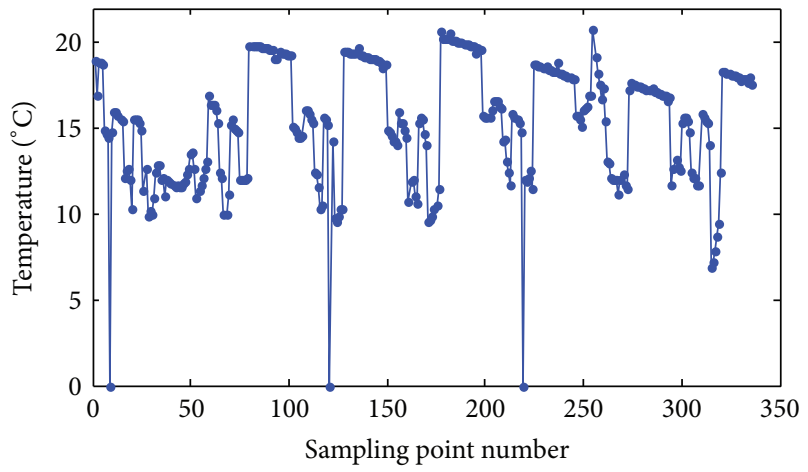

(c)

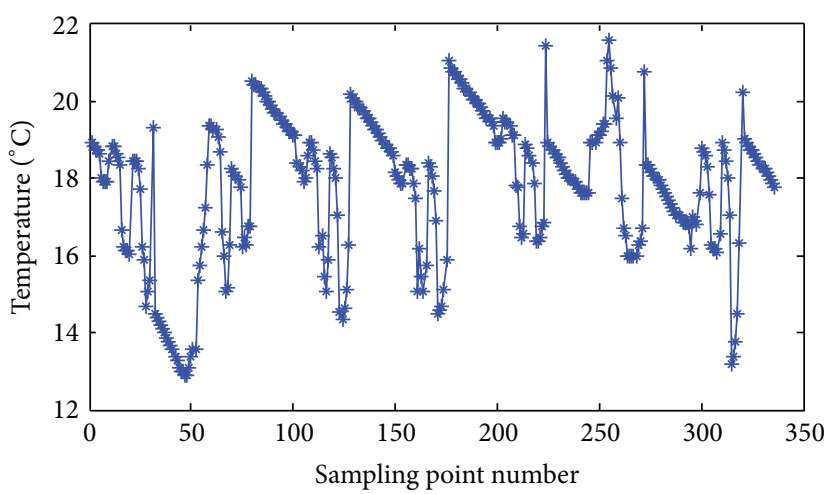

(b)

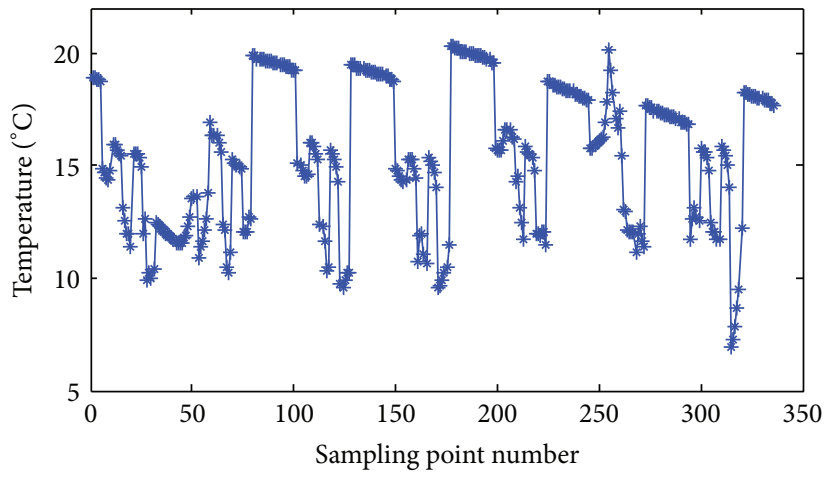

(d)

FIGURE 4: Comparison of CVs and IRVs for water temperatures. (a) CVs of supply water; (b) IRVs of supply water; (c) CVs of return water; (d) IRVs of return water.

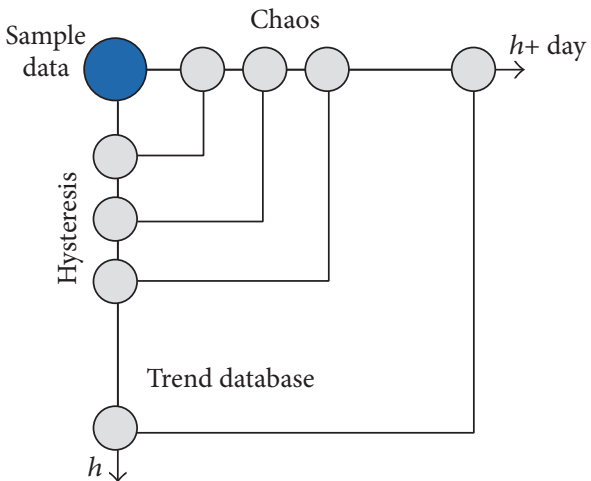

FIGURE 5: Schematic diagram of data variation direction.

TABLE 2: Summary of chillers energy consumption data.

\begin{tabular}{lcc}
\hline Start time & End time & Quantity \\
\hline 2014-07-22, 23:30 & $2014-07-28,13: 30$ & 268 \\
$2014-07-28,14: 00$ & $2014-08-15,00: 30$ & 838 \\
$2014-08-15,15: 30$ & $2016-06-21,14: 30$ & 33262 \\
$2016-02-27,15: 00$ & $2016-08-20,04: 30$ & 8280 \\
\hline & Total & 42648 \\
\hline
\end{tabular}

chaos characteristic of water temperature [24]. Generally, if the chilled water system has higher nonlinearity, the chaos characteristic may become more obvious. Chaos value can be calculated using

$$
C=\frac{1}{n}\left|\sum_{k=1}^{n} \frac{\left(T_{\mathrm{IRV}} \mid k+T\right)-\left(T_{\mathrm{IRV}} \mid k\right)}{\left(T_{\mathrm{IRV}} \mid k\right)}\right|,
$$

where $T_{\text {IRV }} \mid k$ is IRV of water temperature in the sampling point $\left({ }^{\circ} \mathrm{C}\right) ; T_{\text {IRV }} \mid k+T$ is IRV of water temperature in the adjacent cycle sampling point $\left({ }^{\circ} \mathrm{C}\right)$.

3.2.2. Ordinate: Hysteresis of Water Temperature. Not only does the current input value determine the output value of chilled water system, but also the variation tendency (VT) will produce a certain influence. For instance, VT of the outlet water temperature of $1 \#$ chiller in one day can be divided into three intervals: rising $(\uparrow)$, declining $(\downarrow)$, and constant $(\rightarrow)$, as shown in Table 3. According to the analysis and the statistics of the operating data, the sampling time is divided into seven intervals (including probability (Pro) and average slope (A_Slope) when these conditions happen). Therefore, the system has different output values while the input has the same set value in the water temperature rising and declining processes; this phenomenon is recognized as hysteresis. Thus, 


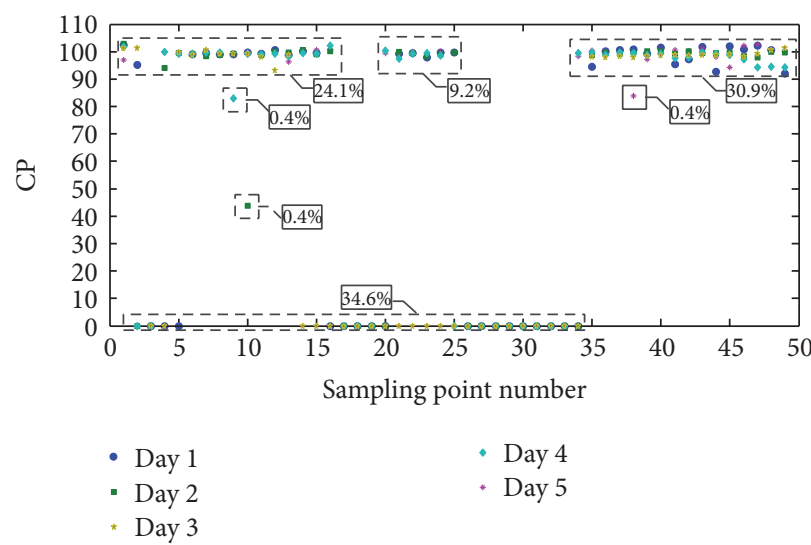

FIGURE 6: CP distribution of 1 \# chiller in five days.

TABLE 3: Intervals of outlet water temperature of 1 \# chiller.

\begin{tabular}{lcccccccc}
\hline Interval & $0: 00-01: 00$ & $1: 00-01: 30$ & $1: 30-05: 30$ & $5: 30-10: 00$ & $10: 00-11: 00$ & $11: 00-14: 00$ & $14: 00-18: 30$ & $18: 30-0: 00$ \\
\hline VT & $\uparrow$ & $\downarrow$ & $\uparrow$ & $\downarrow$ & $\uparrow$ & $\downarrow$ & $\uparrow$ & $\rightarrow$ \\
Pro (\%) & 87.2 & 86.8 & 90.5 & 88.3 & 75.7 & 83.6 & 80.5 \\
A_Slope & $0.12 \sim 0.33$ & $1.63 \sim 2.57$ & $1.3 \sim 3.2$ & $2.01 \sim 2.62$ & $3.42 \sim 4.16$ & $1.08 \sim 2.19$ & $2.66 \sim 3.71$ & $0.03 \sim 0.06$ \\
\hline
\end{tabular}

the relationship between input and output is not simply mapping. The hysteresis value can be calculated using

$$
H=\frac{1}{n}\left|\sum_{i}^{n}\left\{\left(T_{\mathrm{IRV}}(i) \mid R\right)-\left(T_{\mathrm{IRV}}(i) \mid D\right)\right\}\right|,
$$

where $T_{\text {IRV }}(k) \mid R$ is collection value in temperature rising processes $\left({ }^{\circ} \mathrm{C}\right) ; T_{\mathrm{IRV}}(k) \mid D$ is collection value in temperature falling processes $\left({ }^{\circ} \mathrm{C}\right)$.

3.2.3. Random Chillers CP. Chiller provides the required cooling capacity for terminal devices. Since the cooling and heating loads vary randomly, the chiller operates randomly as well. If designers use the partial load ratio to reflect the operation characters of chiller, various sensors should be installed in the indoor and outdoor environment, and the process is quite complicated. The most simple and economical method is using CP to replace the partial load ratio, and the chiller is controlled sequentially. The chiller power can be calculated using (13). Figure 6 shows the current distribution and percent of sampling point in five days; the chiller operates usually under partial load and CP value is random.

$$
E=t \times \mathrm{CP} \times P
$$

Both the fixed frequency and the variable frequency chillers have different CP in the adjacent days. For describing the random value, average value is used as the evaluation criterion; it can be written as the following equation:

$$
R=\frac{1}{n} \sum_{i}^{n} \frac{\mathrm{CP}(i)-\overline{\mathrm{CP}}}{\overline{\mathrm{CP}}}
$$

3.2.4. Case Study. Figure 7 shows the percentages of each average index of CP and chilled water temperatures using the collected data in both July and August. The chaos amplitudes of the supply and the return chilled water increase, indicating that the load fluctuation of CWL gets serious with the rising outside temperature in August. However, the hysteresis amplitudes of the supply and the return chilled water fluctuation vary within a narrow range, which shows the water temperature variation is unconspicuous when the same set value are in both the rising and falling processes. The radar chart analytical method is a comprehensive assessment and multivariate analysis technology. This method especially suits the object of multiple attribute description and can make an overall evaluation. Using the radar chart, the status of the evaluation objects can be seen intuitively. Figures 7(c) and $7(d)$ show the quota distribution of nonlinear phenomena in the adjacent days. According to the analysis, chaos characteristics and randomness are the largely influenced nonlinear index, and the development tendency of these characteristics can be predicted using NVTD.

\section{Data Model}

Artificial neural network processes the information by imitating the biological neural network. It is a mathematical model based on the physiological research results about the brain, and often simulates some mechanisms to achieve the prediction function for the performance and parameters in air conditioning systems. The major training method is guided learning, which is able to adjust parameters according to the training sample and make the output value of network close to the known samples. There is not any specified network structure or learning algorithm suitable for all issues, and it is necessary to improve the network structure for 


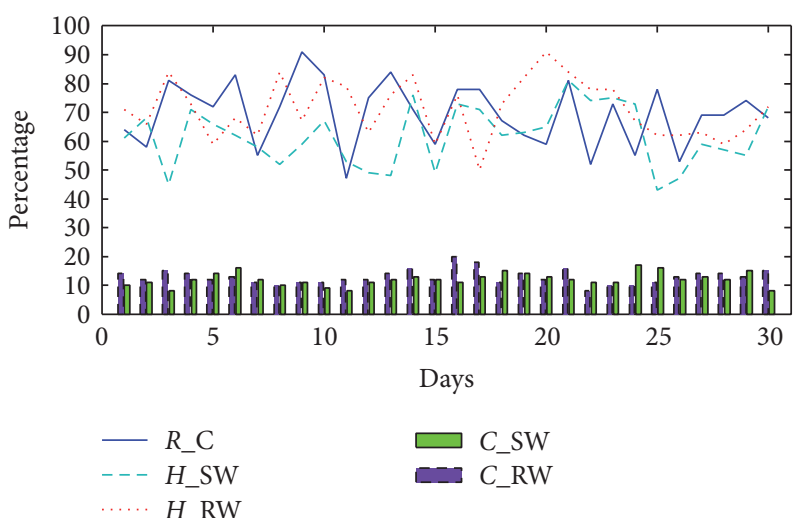

(a)

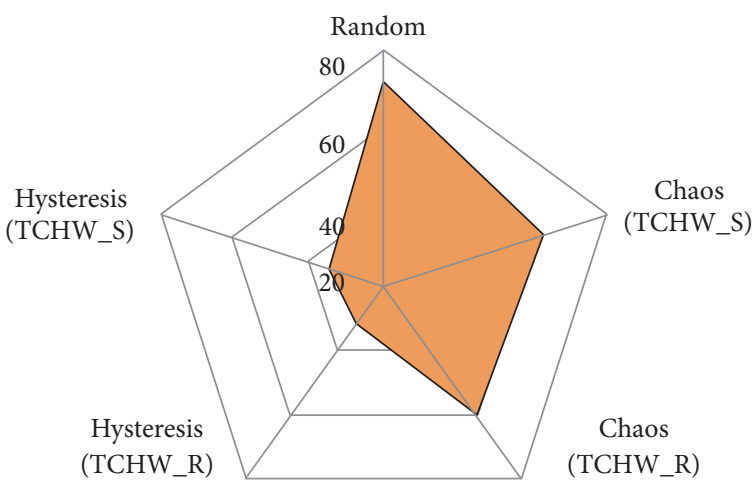

(c)

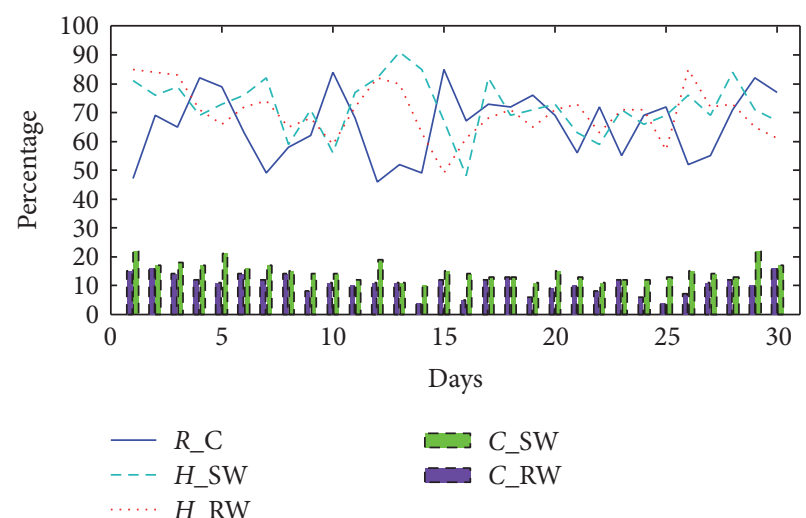

(b)

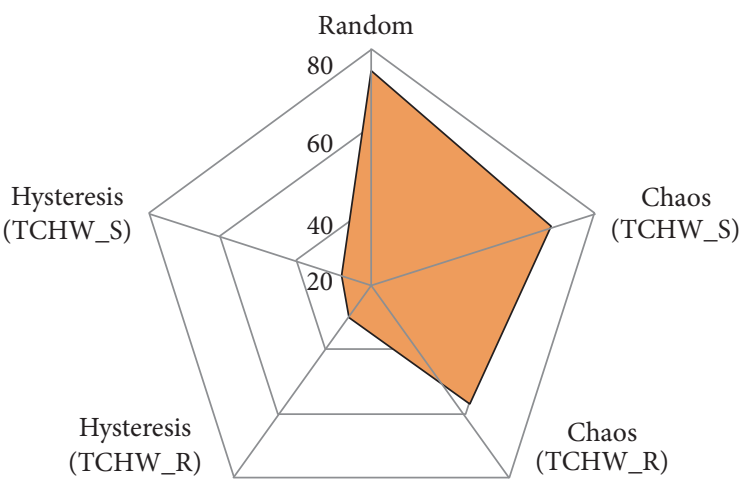

(d)

Figure 7: Average nonlinear phenomena index in two months and days. (a) July; (b) August; (c) Day 1; (d) Day 2; R_C, randomness value of 1\# chiller and 2\# chiller; $H \_S W$, hysteresis value of supply water; $H \_R W$, hysteresis value of return water; $C \_S W$, chaos value of supply water; C_RW, chaos value of return water.

specific issues. In this paper, the evaluation (Eva) layer based on NVTD is added to the Elman and BP networks.

The function of the evaluation layer is to indicate the nonlinear trends during the modeling process and to judge the output of the model. The output value of the hidden layer should meet the corresponding nonlinear trends value. Further, evaluation layer reduces the deviation between the predicted value and the actual value and enhances the calculation pertinence in the big fluctuation region.

4.1. Chiller Operation Model. Elman neural network structure includes input layer, hidden layer, connection layer, and output layer $[25,26]$. The transfer functions of the output layer and the hidden layer are linear and nonlinear functions, respectively. The transmission function of the connection layer is linear and acts as the input of hidden layer together with the network input, so this network can achieve the dynamic memory. In this paper, the structure of Elman neural network is combined with the evaluating layer of the nonlinear trend. The form of improved Elman neural network (N-Elman) is 5-2-2-5-2 (5 input neurons, 2 connection neurons, 2 hidden neurons, 5 Eva neurons, and 2 output neurons) as shown in Figure 8.
4.1.1. Data Selection Rule of N-Elman. During the training cycle, every 6-day data is used to update the $\mathrm{CP}$ model as the training pattern, while every 5-day data is selected as the input vector. Data in the 6th day is selected as the target vector, while data in the 7th day is selected as the test data. Figure 9 shows the data training rule, and the chiller operation model will stop the calculation until meeting the requirements.

4.1.2. Network Formula of N-Elman. In the hidden layer, the output in network can be calculated using

$$
\begin{aligned}
h_{\mathrm{CP}}(k)=f\left(\omega^{I 1} y_{c}(k)+\omega^{I 2} \mathrm{CP}\left(k^{\prime}\right)\right) \\
k^{\prime} \in(k-1, k-2, \ldots, k-5),
\end{aligned}
$$

where

$$
f=\frac{1}{1+e^{x}}
$$

In the evaluation layer, it is applied to approximate the value of nonlinear phenomena indicator function. The output target can be calculated using

$$
M_{\mathrm{CP}(i)}(k)=R_{i}(k) .
$$




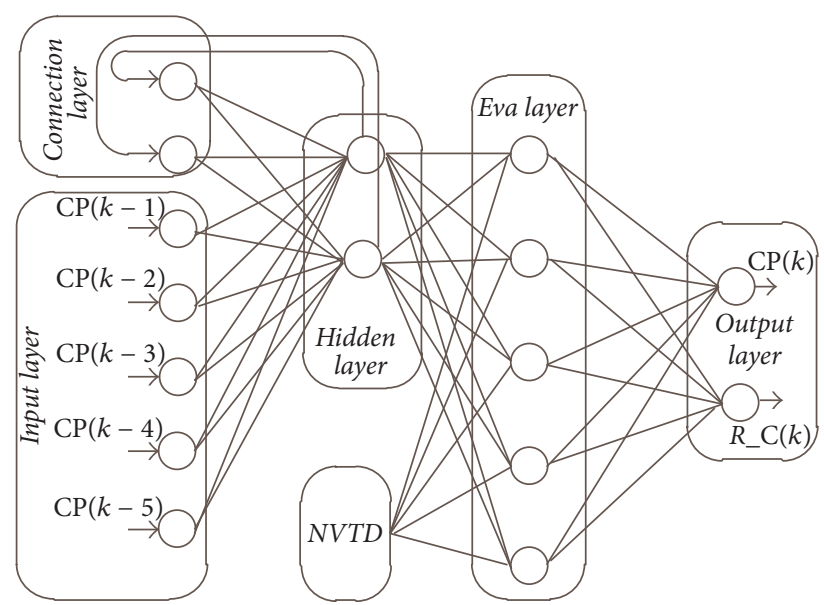

Figure 8: The structure of N-Elman model.

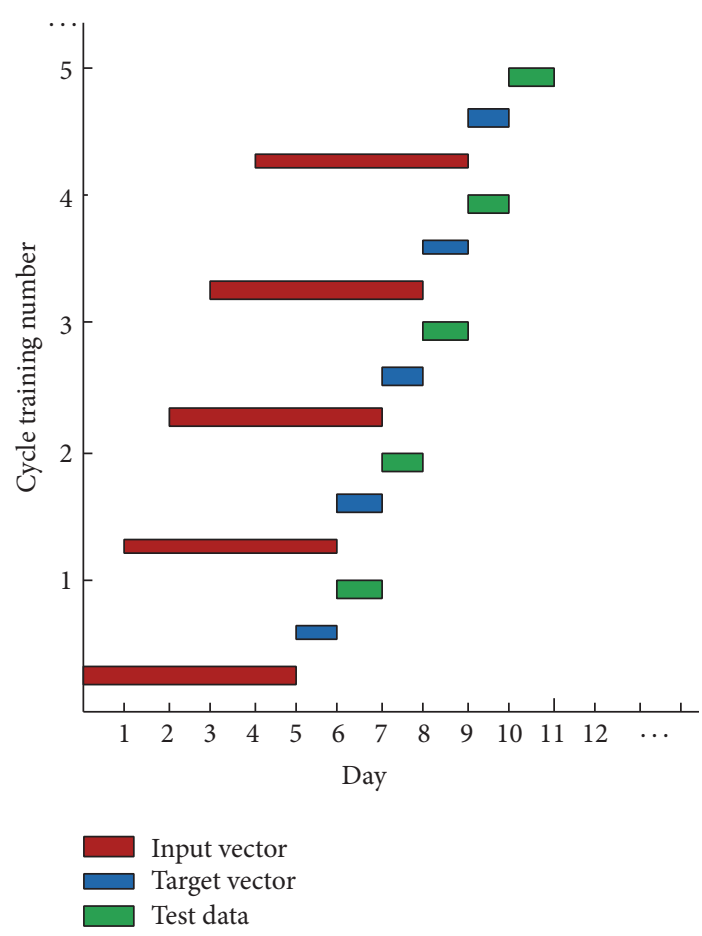

FIGURE 9: Data training rule.

The output of evaluation layer can be calculated using

$$
O_{\mathrm{CP}(i)}(k)=W_{\mathrm{CP}(i)}^{T} \sigma\left(V_{\mathrm{CP}(i)}^{T} z(k)\right) .
$$
using

The error function of evaluation layer can be calculated

$$
e_{\mathrm{CP}(i)}(k)=O_{\mathrm{CP}(i)}(k)-M_{\mathrm{CP}(i)}(k) .
$$

The minimize objective function of evaluation layer can be calculated using (20), and weights can be updated using (21) (22).

$$
\begin{gathered}
E_{\mathrm{CP}(i)}(k)=\frac{1}{2} e_{\mathrm{CP}(i)}^{T}(k) e_{\mathrm{CP}(i)}(k), \\
W_{\mathrm{CP}(i)}(j+1)=W_{\mathrm{CP}(i)}(j)-\alpha_{\mathrm{CP}}(j)\left[\frac{E_{\mathrm{CP}(i)}(k)}{W_{\mathrm{CP}(i)}}\right], \\
I_{\mathrm{CP}(i)}(j+1)=P_{\mathrm{CP}(i)}(j)-\alpha_{\mathrm{CP}}(j)\left[\frac{E_{\mathrm{CP}(i)}(k)}{P_{\mathrm{CP}(i)}}\right] .
\end{gathered}
$$

In the output layer, $\mathrm{CP}(k)$ can be calculated using

$$
\mathrm{CP}(k)=g\left(\omega^{I 3} h_{\mathrm{CP}}(k)\right) .
$$

The objective function can be calculated using

$$
N(k)=\frac{1}{2}\left(\mathrm{CP}_{d}(k)-\mathrm{CP}(k)\right)^{T}\left(\mathrm{CP}_{d}(k)-\mathrm{CP}(k)\right) .
$$

4.2. Chilled Water Temperature Model. BP neural network is a multilayer feed-forward network, including input layer, hidden layer, and output layer. Among them, the number of hidden layers can be adjusted according to the requirements of designers. BP neural network is widely used for predicting parameters in air conditioning systems [27]. It has been proven that BP neural network can realize any complex nonlinear mapping and is particularly suitable for solving problems with complex inner mechanisms. However, some localized problems are difficult to be solved in the counting process of BP neural network. Therefore, the BP network structure in most studies is often improved according to the actual situation. For enhancing the accuracy of predicted value and avoiding the localized problems, the improved $\mathrm{BP}$ model adds the Eva layer combining with NVTD. The form of improved BP neural network (N-BP) is 6-10-6-3 (6 input neurons, 10 hidden neurons, 6 Eva neurons, and 3 output neurons), as shown in Figure 10.

4.2.1. Data Selection Rules of N-BP. If data in a sample has the bad relevance, it is hard for the network to predict the performance. Therefore, it is very rigorous to select the parameters. In this case, the correlation between the supply water temperature and the return water temperature is close. In order to carry out the network calculation, N-BP adopts 5856 data patterns of chilled water temperature. $80 \%$ of them are used for training and $10 \%$ are used for verifying, while the other $10 \%$ are used for testing.

4.2.2. Network Formula of N-BP. Chaos and hysteresis of chilled water temperatures from NVTD are selected as constraint conditions for the network output. The input layer includes TCHWS and TCHWR; $C_{w}$ will be in the range from $70 \%$ to $85 \%$ and $H_{w}$ will be in the range from $10 \%$ to $15 \%$ in evaluation layer according to the data from NVTD. 


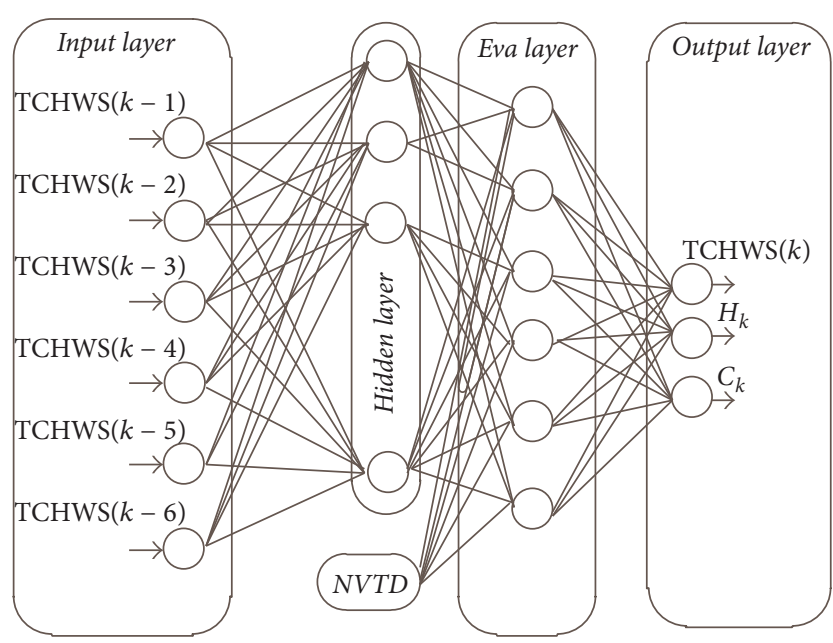

(a)

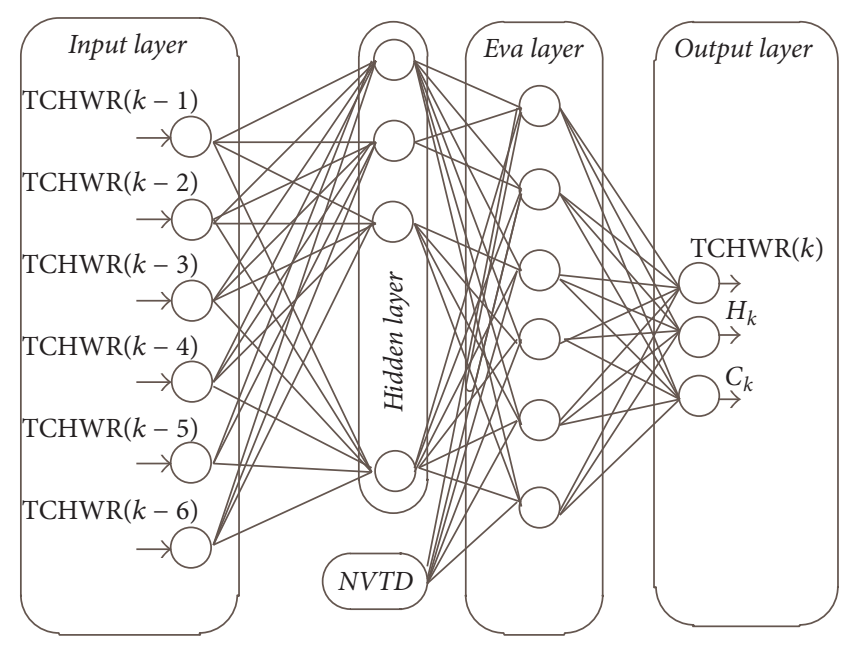

(b)

FIgURE 10: The structure of N-BP models. (a) Supply water temperature; (b) return water temperature.

TCHWS and TCHWR in hidden layer can be calculated using

$$
\begin{aligned}
h_{s} & =f_{1}\left(\left(w_{1 l} \times \operatorname{TCHWS}(k)+w_{2 l} \times \operatorname{TCHWS}(k-1)\right.\right. \\
& \left.\left.+w_{3 l} \times \operatorname{TCHWS}(k-2)\right)-\mathrm{th}_{l}\right), \\
h_{r} & =f_{1}\left(\left(w_{4 l} \times \operatorname{TCHWR}(k)+w_{5 l}\right.\right. \\
& \left.\times \operatorname{TCHWR}(k-1)+w_{6 l} \times \operatorname{TCHWR}(k-2)\right) \\
& \left.-\mathrm{th}_{l}\right),
\end{aligned}
$$

where th $\mathrm{t}_{l}$ is nerve cell threshold value in the hidden layer $(l=1,2,3, \ldots, 10)$. In the evaluation layer, the output target of evaluation layer can be calculated using

$$
M_{C_{-} H(i)}(k)=\left[C_{i}(k), H_{i}(k)\right]^{T} .
$$

The evaluation layer output can be calculated using

$$
O_{C_{-} H(i)}(k)=W_{C_{-} H(i)}^{T} \sigma\left(V_{C_{-} H(i)}^{T} z(k)\right) \text {. }
$$

The error function of evaluation layer can be calculated using

$$
e_{C_{-} H(i)}(k)=O_{C_{-} H(i)}(k)-M_{C_{-} H(i)}(k) \text {. }
$$

The minimize objective function of evaluation layer can be calculated using (30), and weights can be updated using (31) (32).

$$
\begin{aligned}
N_{C_{-} H(i)}(k)= & \frac{1}{2} e_{C_{-} H(i+1)}^{T}(k) e_{C_{-} H(i+1)}(k), \\
W_{C_{-} H(i)}(j+1)= & W_{C_{-} H(i)}(j) \\
& -\alpha_{C_{-} H}(j)\left[\frac{E_{C_{-} H(i)}(k)}{W_{C_{-} H(i)}}\right], \\
I_{C_{-} H(i)}(j+1)= & P_{C_{\_} H(i)}(j) \\
& -\alpha_{C_{-} H}(j)\left[\frac{E_{C_{-} H(i+1)}(k)}{P_{C_{-} H(i+1)}}\right] .
\end{aligned}
$$

In the output layer, $\operatorname{TCHWS}(k+1)$ and $\operatorname{TCHWR}(k+1)$ can be calculated using

$$
\begin{aligned}
& \operatorname{TCHWS}(k+1)=f\left(\sum_{l=1}^{l=10} w_{l j} h_{s}-\mathrm{th}_{y 1}\right), \\
& \operatorname{TCHWR}(k+1)=f\left(\sum_{l=1}^{l=10} w_{l j} h_{r}-\mathrm{th}_{y 2}\right),
\end{aligned}
$$

where th ${ }_{y 1}$ and th $\mathrm{y}_{22}$ are nerve cell threshold value in output layer. MSE (Mean Squared Error) is used as evaluation index; it can be calculated using

$$
\text { MSE }=\frac{1}{n} \sum_{1}^{n} E_{i}^{2}=\frac{1}{n} \sum_{1}^{n}\left(\frac{\text { TCHWS }}{R_{i}}-\overline{\left(\frac{\text { TCHWS }}{R_{i}}\right)}\right)^{2} .
$$

\section{Results}

5.1. Evaluation of CP Model. Figure 11 shows the predicted results of $\mathrm{CP}$ for two chillers using the N-Elman network 

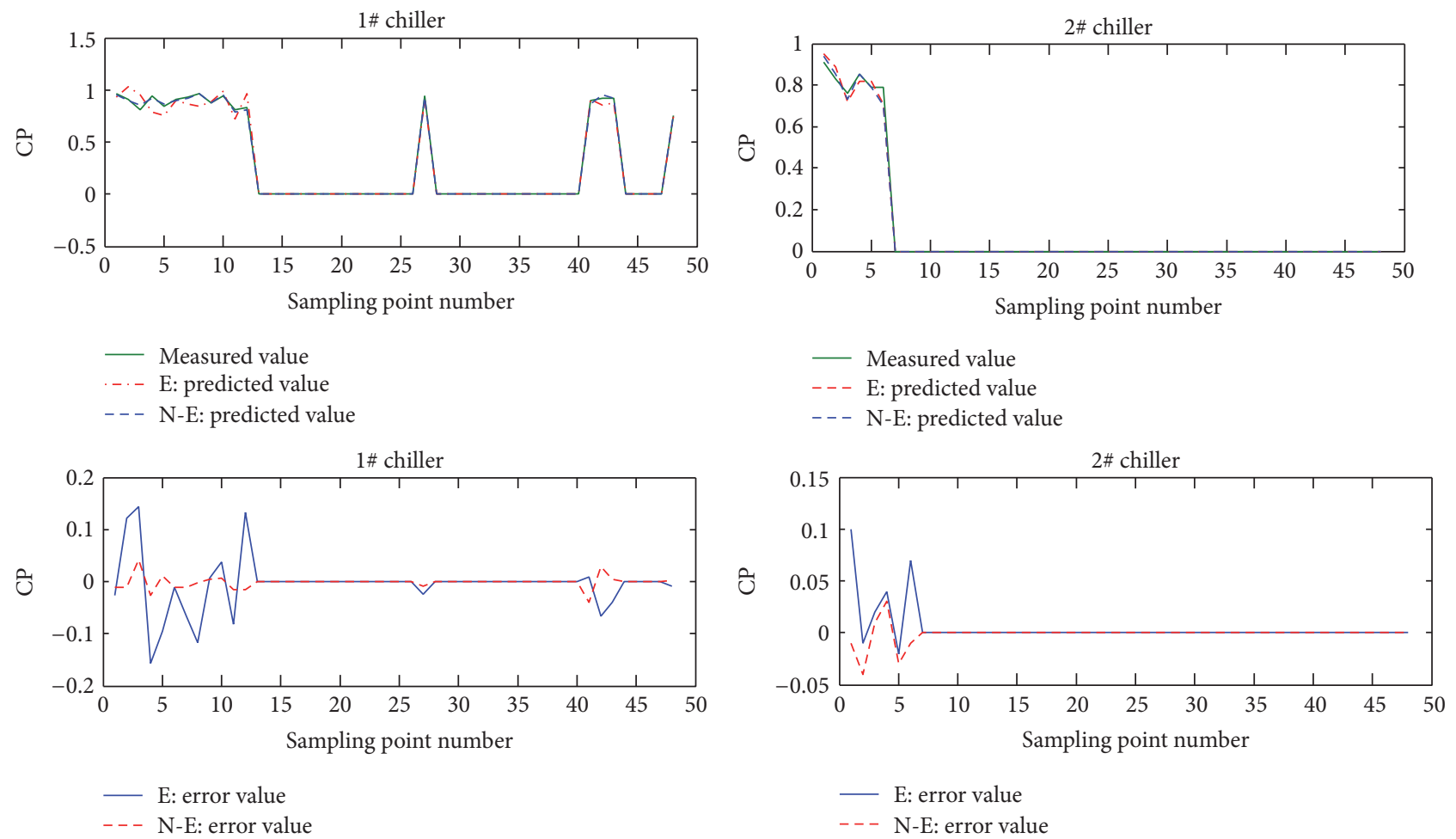

(a)

(b)

FIGURE 11: Comparison of predicted CP values for two chillers using N-Elman and Elman models in one day. (a) 1\# chiller; (b) 2\# chiller.

and the Elman network. In Figure 11, the predicted CP of two chillers (2\# chiller is spare unit) adopting the $\mathrm{N}$ Elman network model is more accurate, and its average errors are less than Elman network model. Table 4 shows the detailed comparisons, where chillers power is derived from (9). Although the iteration of the improved model is increased, the increment is acceptable.

For describing the long-term predictive effect, Figure 12 shows the predictive results of chillers power in 7 days. When the ice storage is melted, the values will be zero. There are 13 anamorphic predicted points using Elman network model in Figure 13(c) and 4 anamorphic predicted points in Figure 13(d) due to the uncertain data between two collection points with less training data near the zero time. However, CP model using the N-Elman overcomes this problem, NVTD provides the variation trend of data, and it is especially suitable for the interval of less data span. In addition, mean absolute errors (MAE) of two chillers using the N-Elman (0.135 and 0.113) are lower than those of using Elman model (0.376 and 0.216$)$. Therefore, the performance of the improved model is more accurate and more stable than Elman model.

5.2. Evaluation of Chilled Water Temperature Model. Figure 13 describes the predicted results for TCHWS and TCHWR of CWL. The predicted performance of N-BP model is more excellent than BP model, with output values more close to the measured values. Table 5 shows the detailed comparisons. The iterations of the modified network are
TABle 4: Comparison of N-Elman and Elman neural network structure performance.

\begin{tabular}{lcccc}
\hline NO & Form & $\overline{E(k)}$ & Power & Iterations \\
\hline 1\# Chiller & Elman & 0.45 & $456.9 \mathrm{~kW}$ & 238 \\
$(462.5 \mathrm{~kW})$ & N-Elman & 0.09 & $462.8 \mathrm{~kW}$ & 387 \\
2\# Chiller & Elman & 0.32 & $158.2 \mathrm{~kW}$ & 127 \\
$(162.7 \mathrm{~kW})$ & N-Elman & 0.05 & $164.1 \mathrm{~kW}$ & 196 \\
\hline
\end{tabular}

TABLE 5: Comparison of N-BP and BP neural network structure performance.

\begin{tabular}{lccc}
\hline NO & Form & MSE & Iterations \\
\hline \multirow{2}{*}{ TCHWS } & BP & 0.21 & 720 \\
& N-BP & 0.17 & 945 \\
\multirow{2}{*}{ TCHWR } & BP & 0.33 & 540 \\
& N-BP & 0.11 & 950 \\
\hline
\end{tabular}

increased, but it reduces the mean square error (MSE) and its value is lower than BP network.

To analyze the long-term performance of improved model, predicted values in 7 days are listed as shown in Figure 14. During the training period, the predicted data of NBP network model are more concentrated than BP network; the reason is that NVTD provides the variation trend of data. Meanwhile, MSEs of TCHWS and TCHWR using NBP model (5.03\% and 3.75\%) are less than BP model (10.19\% 


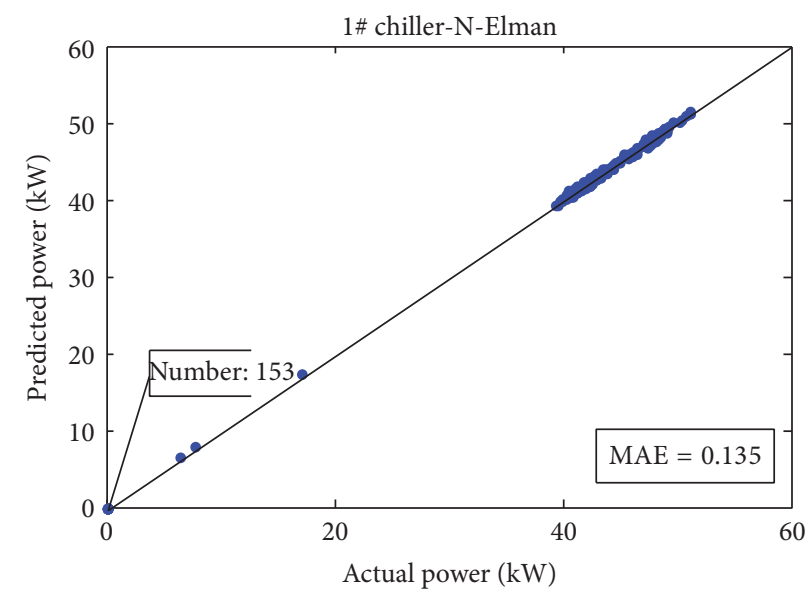

(a)

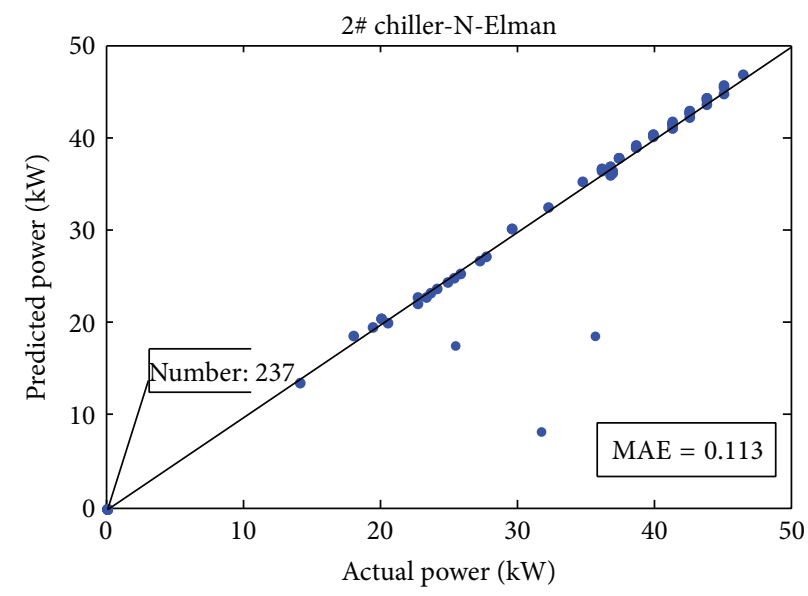

(c)

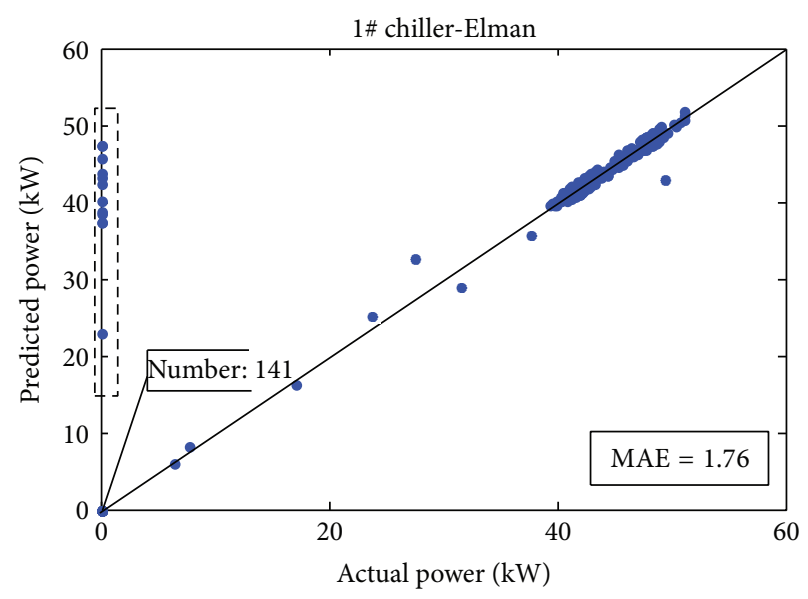

(b)

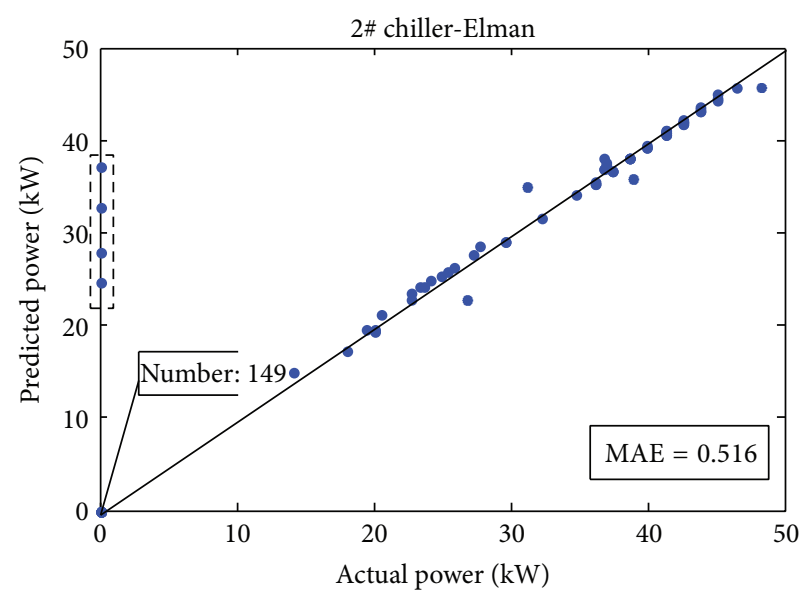

(d)

FIgURE 12: Comparison of predicted values of CP for two chillers using N-Elman and Elman models in 7 days.

and $8.54 \%$ ). So the chilled water temperature with improved model is more accurate and effective than BP model.

5.3. Evaluation of NVTD and Nonlinear Indexes. The major functions of NVTD are providing the learning knowledge of ANN and constraint. For some special operating conditions such as ice storage, there are many sharp variation values (before and after making and melting ice). In this period, the performance of the traditional ANN exists as "jumping value." According to the results of CP model using the NElam network, this phenomenon is effectively avoided. In the training process, either Elman or BP network, often falls into the local optimum because of various data, so the output value should be controlled. According to the comparisons, NVTD has solved this problem using (17) (21) and (26) (31).

Figure 15(a) shows the predicted random index $(R)$ of two chillers. Compared with the Elman model, $R$ evaluated by the N-Elman models $(74.7 \%$ and $64.2 \%)$ are more close to the real value from NVTD. Moreover, Figure 15(b) shows the nonlinear indexes $C$ and $H$ of water temperature using BP and N-BP networks, the predicted index of chaos using BP is lower than the actual value, and hysteresis predicted index is higher than the actual value from NVTD.

\section{Conclusions}

With the increase of sensors in the practical projects, data with high precision for air conditioning system model is required. However, the external and internal nonlinear interferences have great influences, reducing the measurement precision and the data quality. Meanwhile, through excavating data variation trend hiding in collection data from EMCS, optimized parameter prediction data model can be designed. The main goal of this study is to guarantee the accuracy of CWL modeling data using KPOS. This algorithm is derived from recursion using the dynamic state space and is very suitable for multidimensional random estimation. This algorithm also effectively improves the stability and accuracy of the results.

In this study, the data expressions of NVTD are present to analyze the historical data of a case building. Although the predicted values of Elman and BP model are acceptable, improved models (N-E and N-BP) further enhance the predicted performance. Results demonstrate that these improved models acquire more actual operation data compared with Elman and BP model, and they are able to avoid the local 


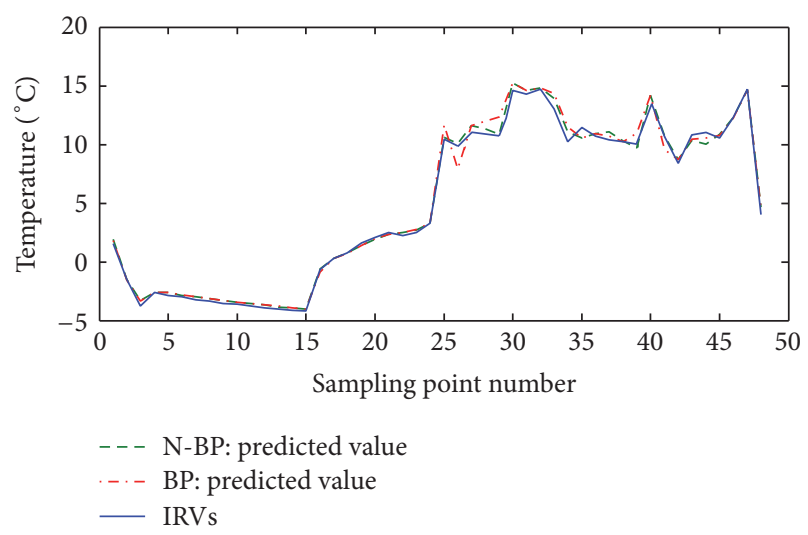

(a)

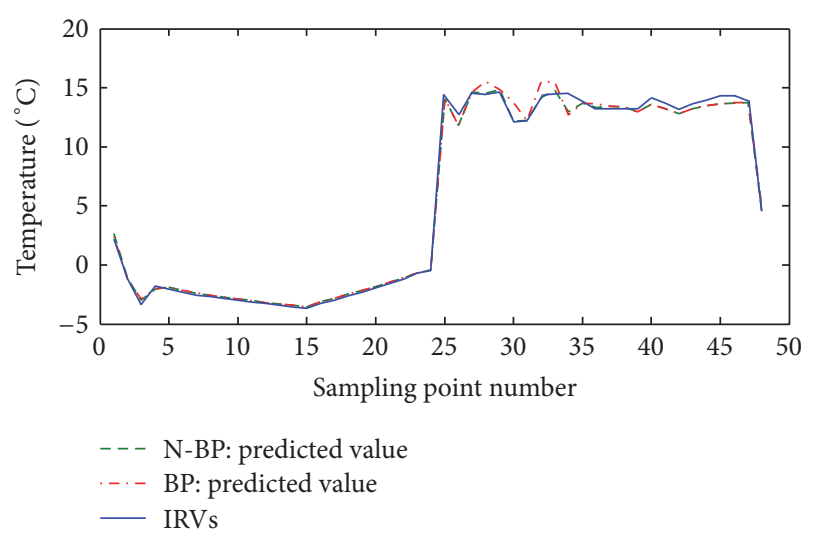

(c)

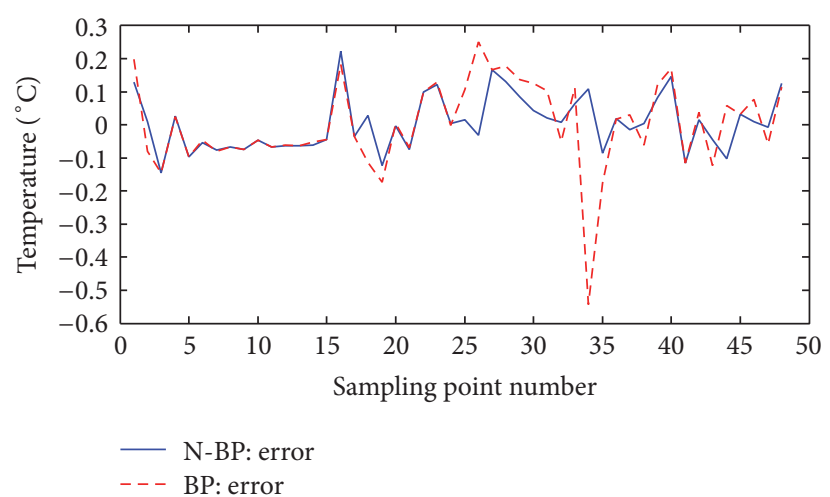

(b)

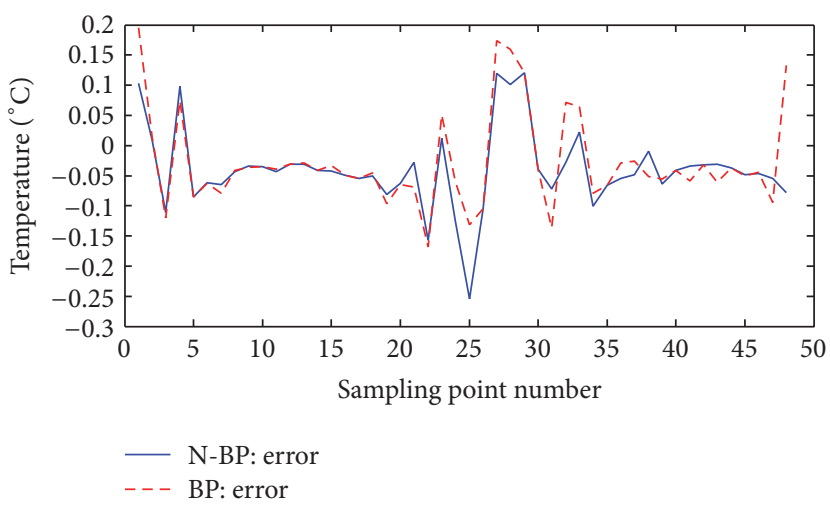

(d)

FIGURE 13: Comparison of predicted values of water temperature using N-BP and BP models in one day; (a) Supply water temperature; (b) error (1); (c) return water temperature; (d) error (2).

optimum value due to the evaluation layer. Therefore, the data processing scheme and model can be regarded as powerful tools for data mining and for increasing the database types. These improved models are also beneficial to the energy distribution and control.

The further study in this field will focus on other parameters (including pressure, flow, and indoor air quality) and designing the controller to reduce the nonlinear influence and energy consumption.

\section{Symbols}

$S_{t}: \quad$ State equation

$\mathrm{O}_{b}$ : Observation equation

$M_{s}$ : State transition matrix

$M_{m}$ : Measurement matrix

$B: \quad$ Input disturbance (value is $Q$ )

$F$ : $\quad$ Observing disturbance (value is $R$ )

$D$ : Deviation function

$G$ : Deviation gain

T: $\quad$ Temperature $\left({ }^{\circ} \mathrm{C}\right)$

$J$ : Fitness function

$c_{1}, c_{2}$ : Learning factors

$r_{1}, r_{2}$ : Random numbers [0 1] $x$ : The position of particle

$C$ : Chaos index

$T$ : Sampling period (h)

$H$ : Hysteresis index

$E$ : Energy consumption of chiller (kW)

$P_{c}$ : Chiller power $(\mathrm{kW})$

$t$ : Operation time (h)

$R$ : Random index

$\overline{\mathrm{CP}}$ : Average CP (\%)

$h$ : Hidden layer

$M$ : Output target

$O$ : Output of evaluation layer

$e$ : Error function of evaluation layer

$W$ : Output matrix of weight

$I$ : Input matrix of weight

P: $\quad$ Primary data $\left(\%,{ }^{\circ} \mathrm{C}\right)$

$g:$ Activation function (Purelin function)

$N$ : Objective function of output layer.

Greek Symbols

$\Gamma$ : Perturbation matrix

$v$ : Particle number $(1,2, \ldots, n)$

$\psi$ : Inertia factor $(\geq 0)$ 


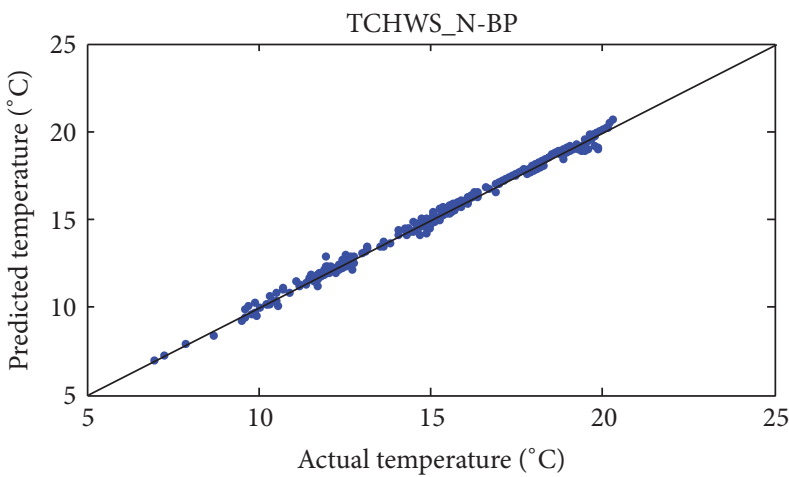

(a)

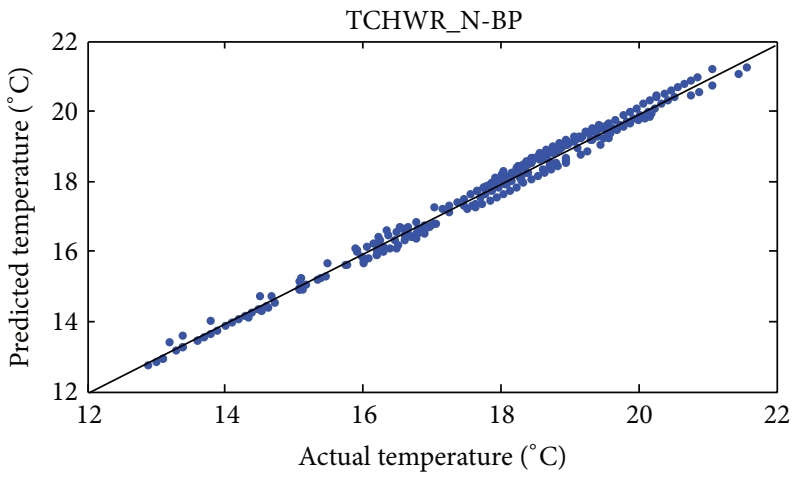

(c)

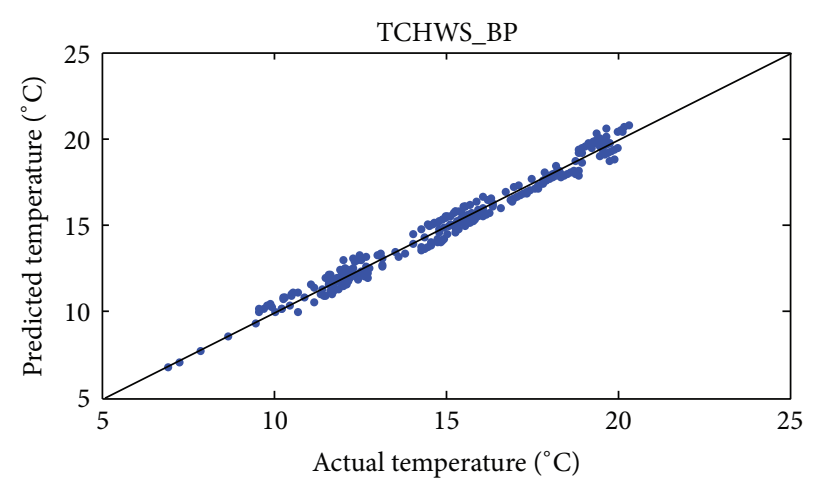

(b)

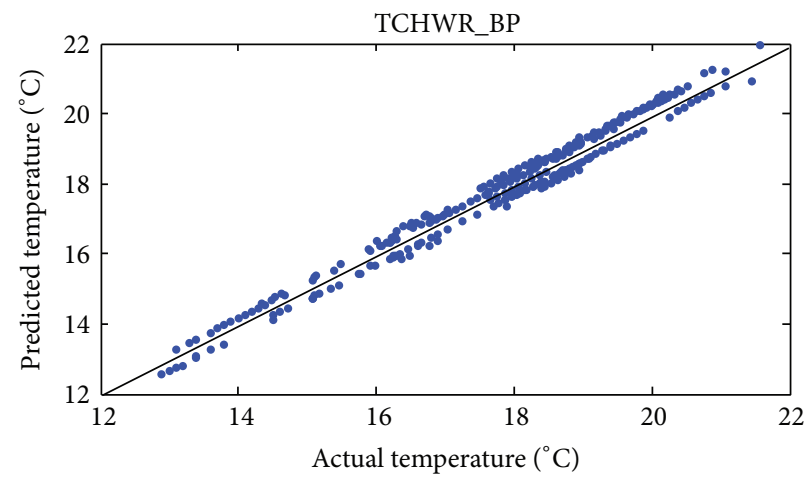

(d)

FIGURE 14: Comparison of predicted values of water temperature using N-BP and BP models in 7 days.

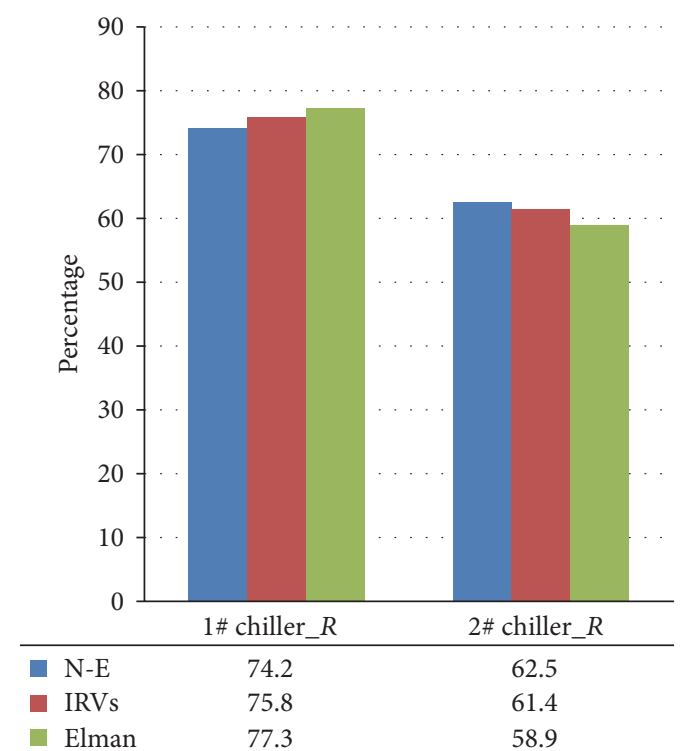

(a)

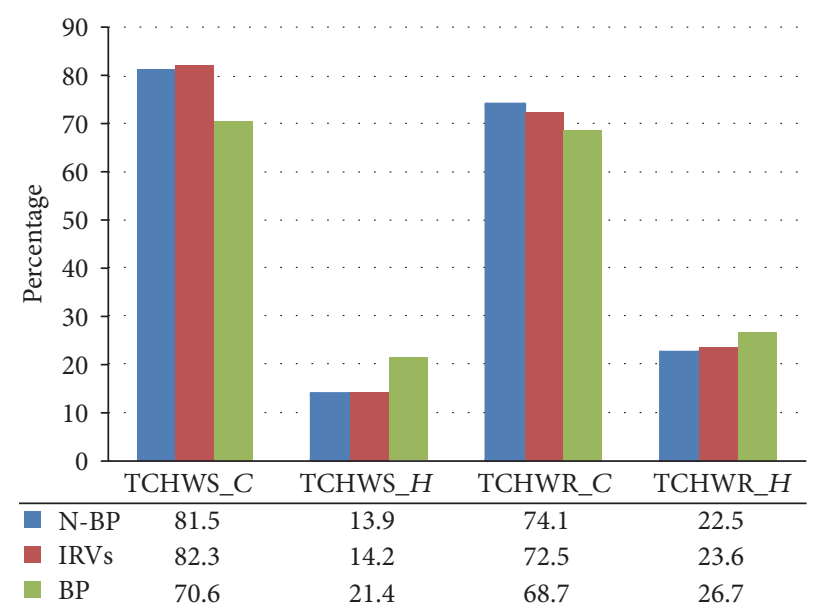

(b)

FIGURE 15: Comparison of predicted values of nonlinear index. (a) Nonlinear index of two chillers using N-E and Elman models; (b) nonlinear index of water temperature using N-BP and BP models. 


\author{
$\lambda$ : Weight factor \\ $\eta$ : Constraint factor \\ $\sigma$ : Activation function \\ $\alpha$ : Learning rate \\ $\omega$ : Connection weight matrix.
}

\section{Superscript}

$d$ : Searching dimensions

$T$ : Transport matrix

I1: Connection weight matrix between associative unit and hidden unit

I2: Connection weight matrix between input unit and hidden unit

I3: Connection weight matrix between hidden unit and output unit.

\section{Subscripts}

IRV: Ideal real value

$s$ : Supply water

$r$ : $\quad$ Return water

il: $\quad$ Neuron connection weight from the input layer to the hidden layer

C_H: Chaos and hysteresis value.

\section{Conflicts of Interest}

The authors declare that they have no conflicts of interest.

\section{References}

[1] S. L. Chang, H. Xie, and S. Zhao, "Data acquisition and control of air-conditioning systems based on virtual instrument technology," Applied Mechanics and Materials, vol. 490-491, pp. 1382-1385, 2014.

[2] Z. Wang, N. Han, and Y. Wang, "Studies on neural network modeling for air conditioning system by using data mining with association analysis," in Proceedings of the International Conference on Internet Computing and Information Services (ICICIS '11), pp. 423-427, Hong Kong, China, September 2011.

[3] A. M. Piette and F. Hannah, "Data mining using HVAC diagnostic tools and EMCS data," Heating, Piping, Air Conditioning Engineering, vol. 74, pp. 30-37, 2002.

[4] Z. Hou, Z. Lian, Y. Yao, and X. Yuan, "Data mining based sensor fault diagnosis and validation for building air conditioning system," Energy Conversion and Management, vol. 47, no. 15-16, pp. 2479-2490, 2006.

[5] T. A. Reddy, L. K. Norford, and W. Kempton, "Shaving residential air-conditioner electricity peaks by intelligent use of the building thermal mass," Energy, vol. 16, no. 7, pp. 1001-1010, 1991.

[6] F. Giuseppe, N. Ettore, L. E. Padovana, and A. Perdichizzia, "Modeling, design and construction of a micro-scale absorption chiller," Energy Procedia, vol. 82, pp. 577-583, 2015.

[7] W.-J. Zhang, C.-L. Zhang, and G.-L. Ding, "Transient modeling of an air-cooled chiller with economized compressor. Part I: model development and validation," Applied Thermal Engineering, vol. 29, no. 11-12, pp. 2396-2402, 2009.
[8] W.-J. Zhang, S.-F. Ding, and C.-L. Zhang, "Transient modeling of an air-cooled chiller with economized compressor. Part II: application to control design," Applied Thermal Engineering, vol. 29, no. 11-12, pp. 2403-2407, 2009.

[9] R. Whalley and A. Abdul-Ameer, "Heating, ventilation and air conditioning system modelling," Building and Environment, vol. 46, no. 3, pp. 643-656, 2011.

[10] L. He, B. Lei, H. Bi, and T. Yu, "Simplified building thermal model used for optimal control of radiant cooling system," Mathematical Problems in Engineering, vol. 2016, Article ID 2976731, 15 pages, 2016.

[11] A. Thosar, A. Patra, and S. Bhattacharyya, "Feedback linearization based control of a variable air volume air conditioning system for cooling applications," ISA Transactions, vol. 47, no. 3, pp. 339-349, 2008.

[12] J. C.-M. Yiu and S. Wang, "Multiple ARMAX modeling scheme for forecasting air conditioning system performance," Energy Conversion and Management, vol. 48, no. 8, pp. 2276-2285, 2007.

[13] X. Xu, G. Huang, H. Liu, L. Chen, and Q. Liu, "The study of the dynamic load forecasting model about air-conditioning system based on the terminal user load," Energy and Buildings, vol. 94, pp. 263-268, 2015.

[14] N. Nassif, S. Moujaes, and M. Zaheeruddin, "Self-tuning dynamic models of HVAC system components," Energy and Buildings, vol. 40, no. 9, pp. 1709-1720, 2008.

[15] R. Ma, N. Yu, and J. Hu, "Application of particle swarm optimization algorithm in the heating system planning problem," The Scientific World Journal, vol. 2013, Article ID 718345, 11 pages, 2013.

[16] H. Bechtler, M. W. Browne, P. K. Bansal, and V. Kecman, "New approach to dynamic modelling of vapour-compression liquid chillers: artificial neural networks," Applied Thermal Engineering, vol. 21, no. 9, pp. 941-953, 2001.

[17] P. Frey, S. Fischer, and H. Drück, "Artificial Neural Network modelling of sorption chillers," Solar Energy, vol. 108, pp. 525537, 2014.

[18] A. Lazrak, F. Boudehenn, S. Bonnot et al., "Development of a dynamic artificial neural network model of an absorption chiller and its experimental validation," Renewable Energy, vol. 86, pp. 1009-1022, 2016.

[19] Z. Hou, Z. Lian, Y. Yao, and X. Yuan, "Cooling-load prediction by the combination of rough set theory and an artificial neuralnetwork based on data-fusion technique," Applied Energy, vol. 83, no. 9, pp. 1033-1046, 2006.

[20] H. J. Manohar, R. Saravanan, and S. Renganarayanan, "Modelling of steam fired double effect vapour absorption chiller using neural network," Energy Conversion and Management, vol. 47, no. 15-16, pp. 2202-2210, 2006.

[21] L. Zhao, L. Shao, and C. Zhang, "Steady-state hybrid modeling of economized screw water chillers using polynomial neural network compressor model," International Journal of Refrigeration, vol. 58, no. 4, pp. 305-313, 2015.

[22] F. Ding, X. Liu, and X. Ma, "Kalman state filtering based least squares iterative parameter estimation for observer canonical state space systems using decomposition," Journal of Computational and Applied Mathematics, vol. 301, pp. 135-143, 2016.

[23] A. A. Nik, F. M. Nejad, and H. Zakeri, "Hybrid PSO and GA approach for optimizing surveyed asphalt pavement inspection units in massive network," Automation in Construction, vol. 71, part 2, pp. 325-345, 2016. 
[24] Q. G. Yi and X. J. Zeng, "Complex dynamics and chaos control of duopoly Bertrand model in Chinese air-conditioning market," Chaos, Solitons and Fractals, vol. 76, pp. 231-237, 2015.

[25] H.-W. Ge, F. Qian, Y.-C. Liang, W.-L. Du, and L. Wang, "Identification and control of nonlinear systems by a dissimilation particle swarm optimization-based Elman neural network," Nonlinear Analysis: Real World Applications, vol. 9, no. 4, pp. 1345-1360, 2008.

[26] C.-H. Lin, "Recurrent modified Elman neural network control of PM synchronous generator system using wind turbine emulator of PM synchronous servo motor drive," International Journal of Electrical Power \& Energy Systems, vol. 52, no. 1, pp. 143-160, 2013.

[27] M. Mohanraj, S. Jayaraj, and C. Muraleedharan, "Applications of artificial neural networks for refrigeration, air-conditioning and heat pump systems-a review," Renewable and Sustainable Energy Reviews, vol. 16, no. 2, pp. 1340-1358, 2012. 


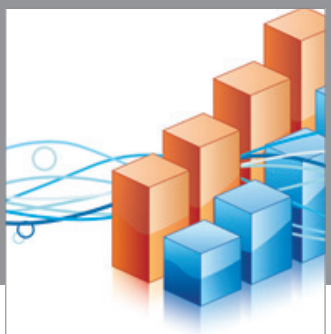

Advances in

Operations Research

vatem alat4

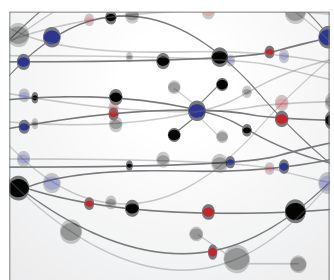

\section{The Scientific} World Journal
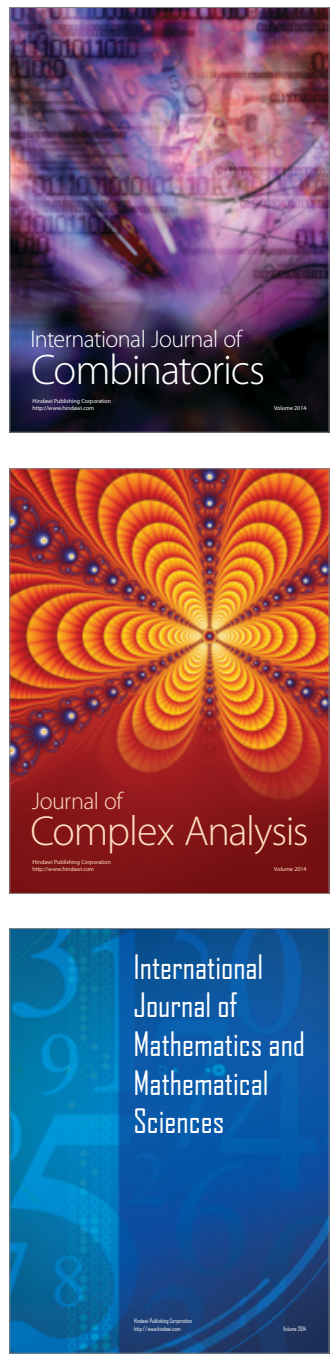
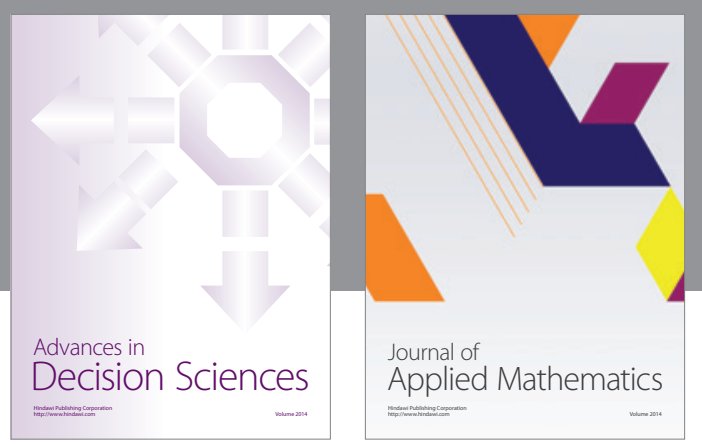

Algebra

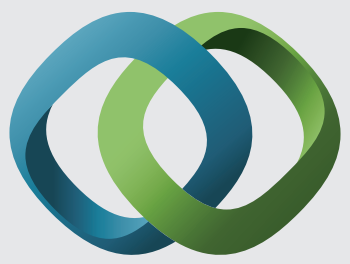

\section{Hindawi}

Submit your manuscripts at

https://www.hindawi.com
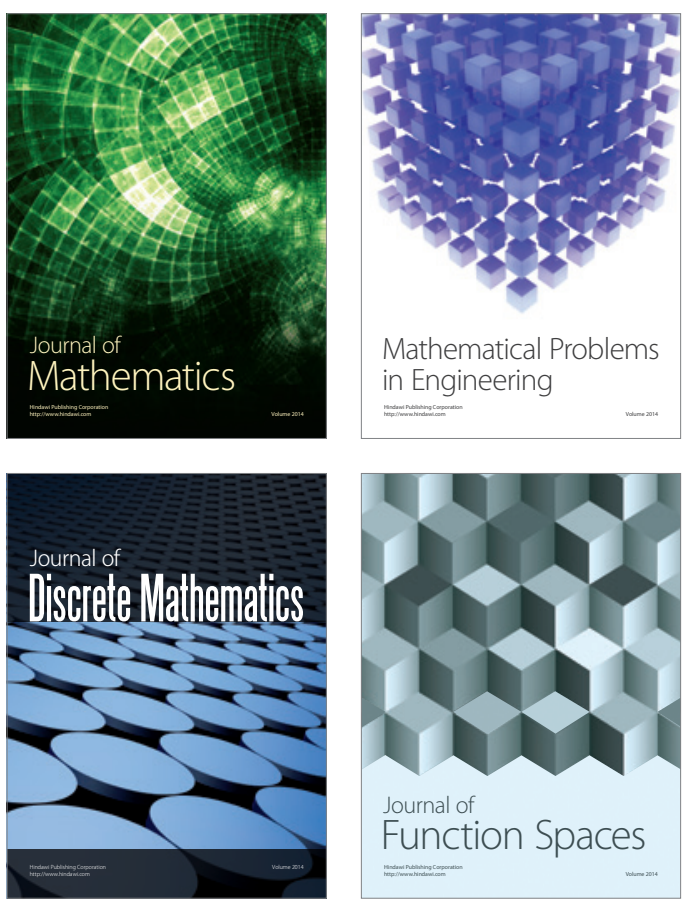

Mathematical Problems in Engineering
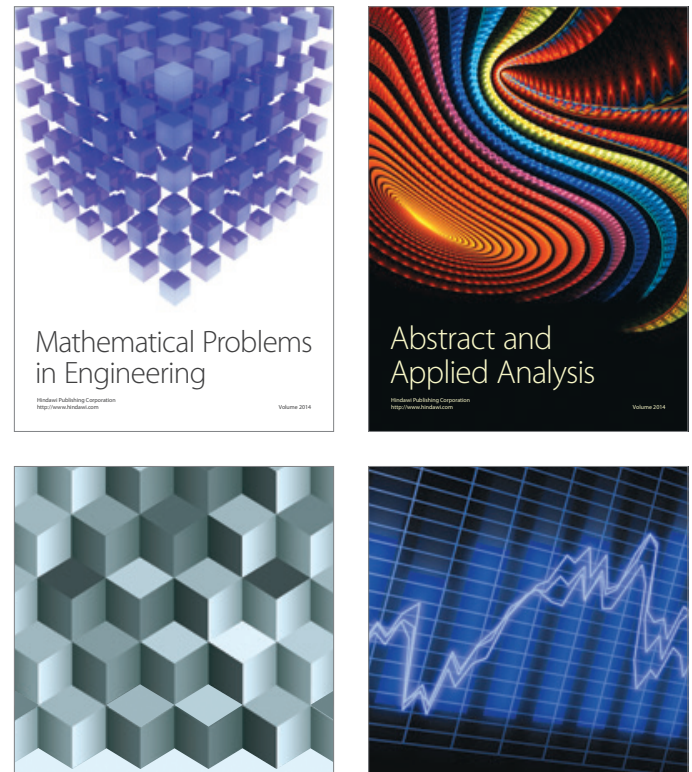

Journal of

Function Spaces

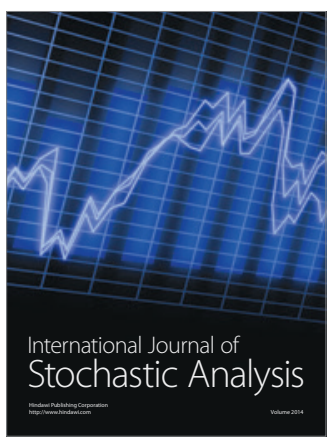

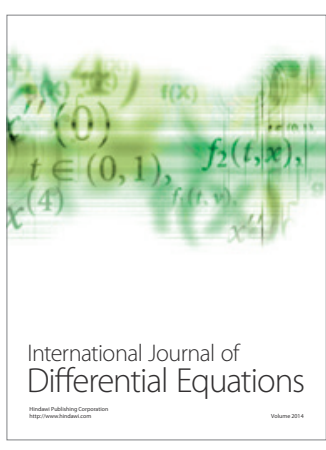
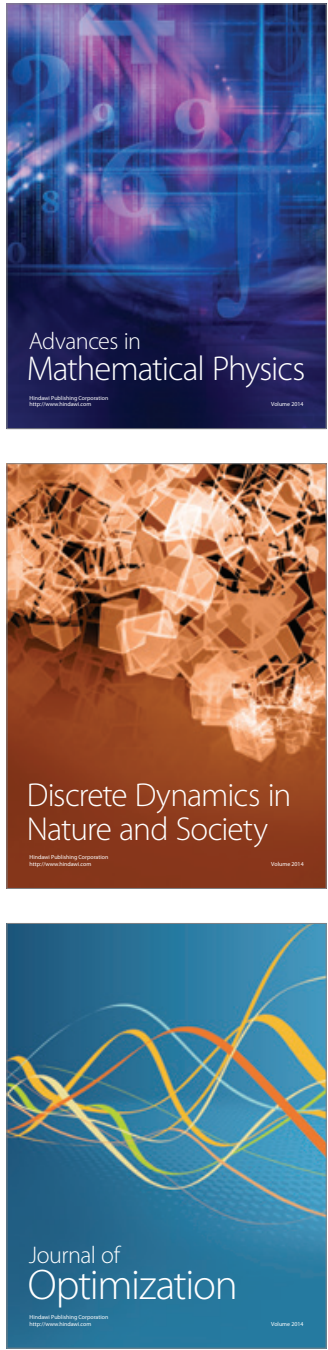